\title{
High-fat dietary consumption promotes disease progression in a surgical joint destabilisation murine model of osteoarthritis: role of systemic eicosanoids and circulating monocytes
}

\section{Anne Elisabeth Kozijn \\ HM de Visser \\ Universitair Medisch Centrum Utrecht}

Universitair Medisch Centrum Utrecht https://orcid.org/0000-0002-5397-4138

MT Tartjiono

TNO Locatie Leiden

F Casbas

University of Nottingham School of Pharmacy

\section{S Ravipati}

University of Nottingham School of Pharmacy

F van der Ham

TNO Locatie Leiden

\section{J Bacardit}

Newcastle University School of Computing Science

\section{J Bouwman}

TNO Locatie Zeist

\section{SC Mastbergen}

Universitair Medisch Centrum Utrecht

\section{AM Zuurmond}

TNO Locatie Leiden

\section{DA Barrett}

University of Nottingham School of Pharmacy

FPJ Lafeber

Universitair Medisch Centrum Utrecht

\section{Bobeldijk}

TNO Locatie Leiden

R Stoop ( $\nabla$ reinout.stoop@tno.nl )

\section{A Mobasheri}

University of Oulu

\section{$H$ Weinans}




\section{Research article}

Keywords: metabolic osteoarthritis, obesity, high-fat diet, inflammation, lipids, eicosanoids, oxylipins, monocytes, macrophages

Posted Date: August 3rd, 2019

DOI: https://doi.org/10.21203/rs.2.12367/v1

License: (a) (i) This work is licensed under a Creative Commons Attribution 4.0 International License. Read Full License 


\section{Abstract}

Background In this study we investigated the contribution of high-fat diet-induced metabolic overload to osteoarthritis (OA) progression originally caused by mild mechanical trauma to the mouse knee joint. We hypothesized that metabolic stress would induce a proinflammatory environment by altering systemic lipid levels and immune cell populations. Methods Twelve-week-old male C57BL/6J mice $(n=20)$ were given a low-fat diet (LFD, $10 \% \mathrm{kcal}$ from fat) or high-fat diet (HFD, $45 \% \mathrm{kcal}$ from fat) for 18 weeks. OA was initiated by transecting the medial meniscotibial ligament of the right knee joint at $t=10$ weeks. OA severity and changes in M1/M2 polarization of synovial macrophage populations were determined in serial coronal FFPE-mounted sections. Eicosanoid levels and monocyte populations were evaluated before and after ligament transection. Results Diet-induced metabolic stress assessed by body weight, systemic cholesterol levels, and insulin resistance index was significantly higher in HFD mice. This group also showed increased cartilage damage, synovitis, and osteophyte formation compared with LFD controls. High-fat feeding elevated systemic arachidonic acid levels, which mainly resulted in increased levels of its cytochrome P450-catalysed diol metabolites 5,6-dihydroxyeicosatrienoic acid (DHET) and 8,9-DHET. High-fat feeding also triggered an increase in pro-inflammatory intermediate CD43++Ly6Cint monocytes after ligament resection. Ligament resection in addition to high-fat feeding induced increased expression of the activation marker CD11c selectively on non-classical CD43++Ly6Clow monocytes. No significant changes in synovial macrophage polarization were observed. Conclusions Metabolic stress resulted in a proinflammatory environment and aggravated injury-induced OA progression. Our results suggest that a CYP450-focused eicosanoid metabolism and activated circulating monocytes may be drivers of this metabolic stress-induced OA progression, contributing to the mechanistic understanding and potentially serving as future diagnostic and prognostic biomarkers for metabolic OA.

\section{Background}

Obesity is the second most significant risk factor for the development of osteoarthritis (OA), the most common form of arthritis[1]. Besides mechanical overload of the joints due to excess body weight, obesity contributes to OA pathogenesis through metabolic disbalance[2], also termed metabolic stress.

Metabolic stress is thought to be a major contributor in a subtype of OA referred to as metabolic OA[3-6], an OA phenotype that is characterized by a systemic low-grade inflammation - although this classification is still under debate[7]. Metabolic OA prevalence is highest in developed countries, where the incidence of obesity progressively increases due to a sedentary lifestyle, low levels of physical activity, and high-caloric diet[8]. The pathogenesis of metabolic $O A$ has been linked to several features of the metabolic syndrome, such as central obesity, insulin resistance and dyslipidemia[2, 9, 10]. Even though the association between $\mathrm{OA}$ and metabolic stress is demonstrated by many studies, the underlying pathophysiologic mechanisms are still vague.

Animal models of diet-induced OA can help elucidate the mechanisms underlying metabolic OA. It is suggested that high-fat feeding induces an overall primed state and the associated metabolic stress undermines the body's capacity to adequately cope with insults like knee injury[11, 12]. The obesity-prone 
C57BL6/J mouse is widely used to study metabolic OA and applied diets are typically high in caloric content, like Western-type diets or high-fat diets (45-60 kcal\% energy from fat)[13]. Diet-induced OA severity is typically mild in animal models provided with a high fat diet only and we found that increased articular cartilage damage compared with low-fat diet (LFD) controls is not assured[14]. Rather, our results suggested that an additional trigger, over and above a high-caloric dietary stressor, is necessary to evoke metabolic OA.

In this study we investigated the contribution of metabolic stress to OA progression induced by mild trauma to the knee joint. We placed C57BL/6J mice on a low-fat diet (LFD, 10kcal\% energy from fat) or high-fat diet (HFD, 45kcal\% energy from fat) and induced OA onset by surgical destabilization of the medial meniscus (DMM). This post-traumatic OA (PTOA) model is low invasive and sufficiently sensitive to study subtle changes in disease progression by mild triggers like genetic background and diet[15, 16]. As obesity is associated with a systemic low-grade inflammation, we hypothesized that diet-induced metabolic stress would induce a proinflammatory environment by altering systemic lipid levels and immune cell populations. We determined systemic eicosanoid levels, lipid mediators best known for their pivotal role in inflammation[17], and circulating monocyte populations. Peripheral blood monocytes are precursor cells for macrophages: an immune cell that plays a role in OA pathogenesis and is known to shift towards the pro-inflammatory (M1) phenotype during adipose-related inflammation[18, 19]. Therefore, we also explored changes in M1/M2 polarization of synovial macrophage populations in PTOA.

\section{Methods}

Please refer to the online supplemental file for a detailed methods section.

The experiment was approved by the institutional Animal Care and Use Committee of TNO and was in compliance with ARRIVE guidelines and European Community specifications regarding the use of laboratory animals. The experiment was carried out in wild-type male C57BL/6J mice purchased from Charles River Laboratories (L'Arbresle Cedex, France) that received a 2-week acclimatization period after transfer to a specific pathogen-free (SPF) animal facility. Twelve-week old male C57BL/6J mice $(n=10$ per group) were given a synthetic high-fat diet (HFD, 45\% kcal from fat) or low-fat diet (LFD, $10 \% \mathrm{kcal}$ from fat) for 18 weeks. Ten weeks after starting the diet, OA was surgically induced in the right knee joint by transecting the medial meniscotibial ligament (MMTL) and destabilizing the medial meniscus (DMM). The left knee joint served as an experimental control and received all surgical procedures except for the MMTL transection (sham surgery). Mice were euthanized 8 weeks post-surgery using gradual-fill $\mathrm{CO}_{2}$ asphyxiation.

Metabolic stress was assessed throughout the experiment by monitoring changes in body weight, body composition, and systemic total cholesterol, glucose and insulin levels. Serial coronal $5 \mu \mathrm{m} \mathrm{FFPE}$ sections were collected throughout the joint at $60 \mu \mathrm{m}$ intervals, stained, and evaluated for cartilage degradation, osteophyte formation and synovitis using the OARSI scoring system specifically designed 
for the mouse[20]. Eicosanoid profiling was performed in fasted plasma samples as described previously[21]. Monocyte subpopulations were analysed in heparin-buffered peripheral blood samples stained with the antibody panel shown in Table 1. Data was obtained using a 3-laser FACSCanto ${ }^{\mathrm{TM}}$ II flow cytometer and analysed with FlowJo v10.2 software.

FFPE sections from DMM-operated knee joints were triple-stained with primary antibodies targeting F4/80 (pan-macrophage), iNOS (M1) and CD206 (M2). Quantification was performed in the lateral patellofemoral synovial lining (ROI), unmixed colour spectra were obtained with a Nuance multispectral imaging system (40x magnification), and analysed with ImageJ $1.51 \mathrm{n}$ software. Statistical analysis was performed using IBM SPSS software. In all analyses, a probability value $<0.05$ was considered statistically significant. Unless stated otherwise, data are presented by the median with interquartile range (range between the 25th to 75th percentiles).

\section{Results}

\section{Metabolic stress aggravates PTOA development}

Diet-induced metabolic stress was observed in the HFD group, as demonstrated by the metabolic profile of the HFD animals at the end of the study (Table 2). Metabolic stress was already present before surgery: body weight, cholesterol levels, and HOMA-IR were significantly increased in HFD mice compared with LFD controls after 9 weeks (data not shown). Body composition analysis revealed that the increase in body weight was completely attributed to an increase in body fat mass.

Articular cartilage damage was assessed by histological examination to evaluate the effect of HFD on DMM-induced cartilage damage. In the sham-operated contralateral knee joints hardly any cartilage damage was observed (Figure 1). DMM surgery on the other hand induced clear OA development in both groups, with diet-induced spatial differences in cartilage damage (Figure 1). Cartilage damage was significantly aggravated in HFD mice compared with controls, with median [interquartile range] OA scores of 19.4 [15.1-20.9] in HFD versus 13.8 [8.0-20.0] in LFD mice $(p=0.049$, Figure $2 A)$. This difference could mainly be attributed to increased damage in the lateral tibiofemoral compartments (Figure 2B). HFD mice showed advanced cartilage erosion in both lateral compartments, extending to $25-50 \%$ of the articular surface (8.0 [4.9-10.0]; $p=0.01)$, while LFD mice showed increased fibrillation, fissures and occasional cartilage loss <25\% (3.8 [3.0-8.0]) (Figure 1). The medial knee compartments, typically more affected in the DMM model, were almost completely devoid of cartilage and reached close to the maximal score of 12 in both the HFD and LFD groups (Figure 2C). Therefore, even if potential diet-induced aggravation had occurred, this could not be inferred from the medial side as these were nearing maximal scores (HFD 11.0 [10.6-11.8]; LFD 10.0 [5.0-12.0]; $p=0.109$ ).

Synovial inflammation was significantly increased and more severe in HFD mice (7 out of 8 mice; 6.5 [5.3-8.5]) compared with LFD mice (4 out of 7 mice; 1.0 [0.0-6.0]; $p=0.04$, Figure 2D). Sham-operated contralateral knees showed no synovial thickening. Increased osteophyte formation was observed in the 
HFD group compared with the LFD controls (Figure 2E). Sham-operated contralateral knee joints showed hardly any osteophyte formation.

\section{Systemic changes in eicosanoid levels}

Eicosanoids can exert both pro- and anti-inflammatory functions and have been implicated in the pathogenesis of a variety of immunometabolic disorders, like obesity ${ }^{13}$ [17]. Eicosanoids are formed from omega-3 (n-3) or n-6 polyunsaturated fatty acid (PUFA) precursors via the cyclooxygenase (COX), lipoxygenase (LOX), and cytochrome P450 pathways (CYP450, Figure 3)[22]. At large, n-6 PUFA-derived eicosanoids exhibit more pro-inflammatory and n-3 PUFA-derived eicosanoids more anti-inflammatory activity. Although a useful framework, this simplistic dichotomy does not cover the complexity of the physiological effects of eicosanoids in vivo[22].

Because of plasma volume limitations and the volume demands of the assay, we were able to reliably detect the levels of 24 eicosanoids. Overall, minor differences were observed between the diet groups. HFD induced a significant increase in arachidonic acid (AA) levels, a n-6 PUFA precursor, with 947 [765$1257]$ in LFD versus 1538 [1230-1935] in HFD mice $(p<0.01)$ at 14 weeks. To find out how this increase affected total eicosanoid metabolism, we evaluated the changes in eicosanoid profiles of the mice before and after DMM surgery. Pathway analysis revealed that the increase in AA levels mainly translated into increased hydroxylation into the fatty acid diols 5,6-dihydroxyeicosatrienoic acid (DHET) and 8,9-DHET (Figure 3). In addition, 5-HETE and 13-OxoODE levels were increased in HFD mice compared with LFD mice. Similar plasma eicosanoid profiles were observed between 9 and 14 weeks (data not shown).

\section{Activation of circulating monocyte subpopulations}

Monocytes are short-lived mononuclear phagocytes that constitute $\sim 10 \%$ of peripheral leukocytes in humans and $\sim 4 \%$ in mice[23]. Current nomenclature divides murine monocytes into three subpopulations based on their surface expression of CD43 and Ly6C receptors[24]: 1) CD43 ${ }^{+}$Ly $6 C^{\text {hi }}$ classical monocytes; 2) $\mathrm{CD} 43^{++}$Ly6 $6 \mathrm{C}^{\text {int }}$ intermediate monocytes; 3) $\mathrm{CD} 43^{++}$Ly $6 \mathrm{C}^{\text {low }}$ non-classical monocytes. The three subpopulations share a developmental relationship (from classical via intermediate to non-classical) and are phenotypically and functionally different.

Monocyte subpopulation percentages showed subtle differences between the LFD and HFD groups over time (Figure 4A). The most pronounced effect of long-term HFD was an increase in circulating $\mathrm{CD} 43^{++}{ }^{+1} 6 \mathrm{C}^{\text {int }}$ intermediate monocytes at $\mathrm{t}=17$ weeks, at the expense of the $\mathrm{CD} 43^{++}$Ly $6 \mathrm{C}^{\text {low }}$ non-classical subpopulation. This increase showed significance within the total $C D 115^{+} \mathrm{CD} 11 \mathrm{~b}^{+} \mathrm{SSC}-\mathrm{A}^{\text {low }}$ monocyte fraction ( $p=0.044$, data not shown), but due to interindividual variability did not reach significance within the total leukocyte fraction ( $p=0.107$, Figure 4B). 
The increase in intermediate monocytes concurred with a significantly increased expression of the monocyte activation marker CD11c in HFD mice compared with LFD controls (0.8 [0.7-1.4] versus 3.0 [1.63.9] at 9 weeks and 3.0 [1.9-3.4] versus 6.6 [4.6-7.7] at 17 weeks, respectively; Figure 4C). CD11c contributes to monocyte arrest on endothelial cells and, as such, is upregulated by activated monocytes during chemotaxis. Both high-fat diet and knee injury contributed to the increased expression of CD11c, respectively, as: 1) HFD mice showed significantly more CD11c expression compared with LFD controls at 9 and 17 weeks $(p<0.01)$ and 2) LFD mice showed increased CD11c expression over time $(p=0.03)$. Backgating (i.e. highlighting the final gated population within the population of its ancestors) of the $\mathrm{CD} 11 \mathrm{c}^{+}$cells into the total $\mathrm{CD} 115^{+} \mathrm{CD} 11 \mathrm{~b}^{+} \mathrm{SSC}-\mathrm{A}^{\mathrm{low}}$ monocyte fraction revealed that this integrin was predominantly upregulated by the $\mathrm{CD} 43^{++} \mathrm{Ly} 6 \mathrm{C}^{\mathrm{low}}$ non-classical monocytes (Figure 4D).

\section{High-fat feeding induced no macrophage polarization}

As activated monocytes may differentiate into macrophages upon tissue entry, the effects of the HFD on this immune cell type were investigated locally by evaluating the surface area percentage positivity of M1 (iNOS) and M2 (CD206) macrophage markers in the synovial lining of DMM-operated right knee joints.

Synovial thickening was increased in knee joints of HFD mice compared with LFD mice (Figure 5A). The ratio of the surface area percentages between M1 marker iNOS and M2 marker CD206 showed an increase in the HFD mice that did not reach significance (LFD 0.54 [0.39-1.34] versus HFD 1.28 [0.621.66]; $p=0.12$; Figure $5 B$ ). Pan-macrophage marker $F 4 / 80$ positivity was predominantly observed in the upper synovial layer and showed a non-significant increase on HFD (0.94 [0.20-2.78] versus 2.49 [1.615.25]; Figure 5C). The subset-specific markers CD206 and iNOS were upregulated in the HFD group compared with LFD group as well, but none of the markers reached a significant difference between both groups. This was also observed within the $\mathrm{F} 4 / 80^{+}$macrophage fraction, where the colocalization surface area percentages of CD206 and iNOS positivity were similar between diet groups (Figure 5D).

Correlation analyses revealed moderate to high positive associations for the total F4/80-stained surface area of the synovial lining with lateral OA degeneration and synovitis scores (Table S2). Both OA scores significantly correlated to the colocalization of the total area positivity of the three macrophage markers as well. Osteophyte scores showed no correlation with any of the macrophage stains. Within the F4/80 fraction, neither subset marker associated with the evaluated OA features.

\section{Conclusions}

In this study, we investigated to what degree metabolic stress aggravated disease progression in a posttraumatic OA (PTOA) model and which mechanisms could be involved. Mice on a HFD showed significantly more cartilage damage upon knee injury compared with those on a LFD. Increased OA severity also manifested itself in enhanced osteophyte formation and synovial inflammation. Modulation of eicosanoid metabolism towards the CYP450 pathway and activation of monocyte subpopulations 
reflected an aggravated state of systemic immune activation. Locally, HFD-induced synovial thickening resulted in an increase in macrophage markers that positively associated with OA severity, but no clear macrophage polarization was observed. These results suggest that systemic changes, triggered by HFDinduced metabolic stress, aggravate injury-induced OA by dysregulating eicosanoid metabolism and by increasing immune activation.

Aggravation of local injury due to metabolic stress was evident from the observed spatial differences in OA features. HFD mice showed more generalized cartilage damage compared to LFD mice, which displayed the medially oriented cartilage damage known to the DMM model[15]. The observed dietinduced disease aggravation confirms results from other PTOA mouse models on a HFD regimen[16, 25] and is in line with faster disease progression reported for obese OA patients[26]. The clear increases in lateral cartilage damage, synovial inflammation, and osteophyte formation compared with LFD mice qualifies the HFD DMM model as a suitable preclinical mouse model to identify players and pathways involved in metabolic OA. As both the internal sham control joints and the LFD animals developed considerably smaller OA features for all measured parameters, we conclude that HFD feeding on its own has limited potential to induce OA but clearly creates a proinflammatory environment leading to aggravation of PTOA progression.

To identify which factors might contribute to a diet-induced proinflammatory environment, we explored changes in systemic eicosanoid levels. In addition to regulating a wide range of physiologic processes[27], eicosanoids can exert both pro- and anti-inflammatory functions[28]. Of note, synovial fluid eicosanoid profiles were not reflected systemically in a rat model of HFD-induced OA, which suggests differential roles of eicosanoids in local versus peripheral compartments[29]. Although local eicosanoid involvement has been reported in OA[30-33], their systemic role in OA pathogenesis is less explored. In our model, HFD sparked a clear increase in systemic arachidonic acid (AA) levels, a n-6 polyunsaturated fatty acid that can trigger a pro-inflammatory cascade. Increased AA hydroxylation by the enzyme soluble epoxide hydrolase (sEH) in the cytochrome P450 pathway generated increased levels of dihydroxyeicosatrienoic acids (DHETs). This probably reflects the diet-induced metabolic stress, as obesity has been shown to induce altered CYP-expression and increased SEH-generated DHET levels in models of metabolic syndrome[34-36]. Accordingly, metabolic syndrome was ameliorated by pharmacological inhibition of $\mathrm{sEH}[37,38]$. A similar dysregulation of CYP-mediated eicosanoid metabolism has also been observed in patients with cardiovascular disease, a metabolic condition that shares pathophysiological similarities with OA[39], for which sEH inhibitors (sEHi) have shown promising therapeutic effects[40]. Recently, increased synovial fluid levels of sEH-generated n-6 PUFAs were positively associated with knee $\mathrm{OA}[41]$, demonstrating a link between eicosanoid metabolism and $\mathrm{OA}$ progression as well. Although initially considered to be inactive EET degradation products, DHETs have been reported to promote CCL2-mediated monocyte chemotaxis in vivo and to restore sEHi-blocked human monocyte migration in vitro[42]. This is particularly interesting, as the CCL2/CCR2 signalling axis has been shown to preferentially mediate monocyte trafficking and promote inflammation and tissue damage in OA[43]. Because circulating monocytes can replenish tissue-resident macrophage populations, 
which are important players in OA pathogenesis, high systemic DHET levels may directly or indirectly influence the progression of $\mathrm{OA}$ through the activation of peripheral monocyte subsets.

To investigate whether peripheral monocyte subsets were indeed affected in our model, we analysed changes in circulating monocyte subsets before and after knee injury. We observed significantly increased monocytic activation, reflected by the upregulation of $\beta_{2}$-integrin CD11c. HFD mice increased monocytic $C D 11 \mathrm{c}$ expression before DMM surgery, demonstrating a dietary effect. It is interesting that LFD mice showed more CD11 $c^{+}$monocytes at end point (8 weeks after DMM) than before DMM surgery. As it seems not likely that $\mathrm{CD} 11 \mathrm{c}$ is a marker for ageing alone, this finding suggests that monocytic CD11c expression partly reflects the consequences of the DMM surgery. However, due to the lack of external sham-operated controls we were not able to verify this possibility in our study. Our finding that CD11c was predominantly expressed by non-classical monocytes is consistent with earlier reports[44] and points to tissue infiltration by this subset. It might be that the increase in the circulating intermediate $\mathrm{CD} 43^{++}$Ly $6 \mathrm{C}^{\text {int }}$ monocyte subpopulation indicates renewing of the non-classical monocyte pool by maturation of peripheral classical monocytes. Alternatively, the increase of this pro-inflammatory monocyte subset might indicate a role in metabolic OA pathogenesis. In humans, intermediate monocytes appear to be the main population to be perturbed in disease conditions[45], including metabolic syndrome, rheumatoid arthritis and atherosclerosis[46-48]. Though preliminary, our observations show consistency with current literature on the role of monocyte subsets in inflammation and OA. Elevated monocytic activation has also been reported in women with knee OA and positively associated with BMI[49]. Similar preclinical findings were made in a mouse model of diet-induced atherosclerosis, in which low-grade inflammation sustained elevated levels of CD11 b ${ }^{+} L y 6 C^{\text {hi }}$ proinflammatory monocytes[50]. Here, similar low-grade inflammation instigated significant in vitro expansion of intermediate monocytes in human peripheral blood mononuclear cells[50]. Taken together, our data add to the current understanding that a systemic dysregulated lipid metabolism and immune activation may be independent yet intertwined processes that contribute to the progression of metabolic OA.

We hypothesized that M1 polarization as seen in obesity might drive local metabolic OA pathogenesis, but no significant increases or shift in synovial macrophage subpopulations were observed. The relative increase of synovial $\mathrm{F} 4 / 80^{+}$macrophages in the HFD group is likely an underestimation, as significant synovial thickening inherently affects surface area percentages in our image analysis. However, despite the low sample numbers with interindividual variability, our novel triple immunostaining provided a clear image of the local macrophage polarization. We observed a mixed pattern of both M1 and M2 macrophage markers together with increased iNOS deposition in the synovia of HFD mice (data not shown). These novel findings are in line with clinical studies showing that activated human macrophages in the OA knee joint express both M1 and M2 markers while displaying a pro-inflammatory cytokine profile[51, 52]. Therefore, the increased iNOS secretion in the HFD group might reflect altered macrophage activation (instead of polarization) and indicate a pro-inflammatory microenvironment in the knee joint. 
In conclusion, we have demonstrated that metabolic stress as applied in this model on its own does not provide sufficient stress for OA development, but clearly aggravated PTOA progression by promoting cartilage damage, synovial inflammation, and osteophyte formation. HFD instigated a proinflammatory environment through systemic changes in lipid metabolism and activation of non-classical monocytes. Based on these results, we propose that a CYP450-focused eicosanoid metabolism and activated circulating monocytes may be drivers of metabolic OA progression. These systemic factors might have future potential as diagnostic and prognostic markers for metabolic $\mathrm{OA}$, consistent with conclusions from a recent clinical study $[53,54]$. For the clinical setting, our results imply that pharmacological inhibition of metabolic stress could benefit obese patients with secondary OA. Specifically, inhibition of soluble epoxide hydrolase could be a promising strategy to attenuate metabolic OA.

\section{List Of Abbreviations}

OA Osteoarthritis

PTOA Post-traumatic osteoarthritis

LFD Low-fat diet (10\%kcal energy from fat)

HFD High-fat diet (45\%kcal energy from fat)

DMM Destabilization of the medial meniscus

MMTL Medial meniscotibial ligament

FFPE Formalin-fixed paraffin-embedded

PUFA Polyunsaturated fatty acid

AA Arachidonic acid

DHET Dihydroxyeicosatrienoic acid

CYP450 Cytochrome P450

sEH Soluble epoxide hydrolase

CD115 Cluster of differentiation 115 (Colony stimulating factor 1 receptor)

CD11b Cluster of differentiation 11b (Integrin alpha M)

SSC-A Side scatter-Area

CD43 Cluster of differentiation 43 (Leukosialin)

Ly6C Lymphocyte antigen 6 complex locus $C$ 
CD11c Cluster of differentiation 11c (Integrin alpha X)

F4/80 EGF-like module-containing mucin-like hormone receptor-like 1 (pan-macrophage marker)

CD206 Cluster of differentiation 206 (mannose receptor, M2 macrophage marker)

iNOS Inducible nitric oxide synthase (M1 macrophage marker)

ROI Region of interest

\section{Declarations}

\section{Consent for publication}

Not applicable.

\section{Availability of data and material}

The datasets used and/or analysed during the current study are available from the corresponding author on reasonable request. The lipidomic dataset is also stored in a phenotype database repository and is available by signing up via https://dashin.eu/interventionstudies/. After receiving credentials and logging in, the study can be accessed via https://dashin.eu/interventionstudies/study/show/39162914 or by searching the study code (HFD_DMM) or study title ("High-fat diet feeding aggravates osteoarthritis progression in a surgical mouse model: role of eicosanoids and circulating monocytes").

\section{Competing interests}

The authors declare that they have no competing interests.

\section{Funding}

This manuscript was supported by funding from the Netherlands Organization for Applied Scientific Research (TNO) and the European Union's Seventh Framework Programme for research, technological development and demonstration under grant agreement No. 305815-D-BOARD. Funders did not have any additional role in the study design, data collection and analysis, decision to publish or preparation of the manuscript. Specific roles of the authors are described in the Authors' contributions section.

\section{Authors' contributions}

AEK, AMZ, IB and RS have designed the experiment. AEK, MT, FC, RS, FH, JB and JB have carried out experimental procedures. AEK has been the primary person responsible for writing the manuscript. HMV, SCM, AMZ, DAB, FPJL, IB, RS, AM and HW were involved in drafting the work or revising it critically for important intellectual content. All authors approved the final version to be published.

\section{Acknowledgements}


We are very grateful to Dr. Melina Daans for sharing her expertise and providing micro-surgical training to AEK. We thank Sabina Bijlsma and Eric Schoen for their continued statistical support. We thank Martien Caspers for his assistance with the phenotype database repository. We thank Jan Lindeman and Marlieke Geerts at the Leiden University Medical Center and for their help with the development of the triple chromogenic immunostaining for macrophages. We thank Anne Schwerk for her enthusiastic assistance concerning the histological part of the research.

\section{References}

1. Murphy LB, Cisternas MG, Greenlund KJ, Giles W, Hannan C, Helmick CG. Defining Arthritis for Public Health Surveillance: Methods and Estimates in Four US Population Health Surveys. Arthritis Care Res Hoboken. 2017;69:356-67.

2. Berenbaum F, Wallace IJ, Lieberman DE, Felson DT. Modern-day environmental factors in the pathogenesis of osteoarthritis. Nat Rev Rheumatol. 2018.

3. Bijlsma JW, Berenbaum F, Lafeber FP. Osteoarthritis: an update with relevance for clinical practice. The Lancet. 2011;377:2115-26.

4. Dell'Isola A, Allan R, Smith SL, Marreiros SS, Steultjens M. Identification of clinical phenotypes in knee osteoarthritis: a systematic review of the literature. BMC Musculoskelet Disord. 2016;17:425.

5. Appleton CT, Hawker GA, Hill CL, Pope JE. Editorial: "Weighing in" on the Framingham Osteoarthritis Study: Measuring Biomechanical and Metabolic Contributions to Osteoarthritis. Arthritis Rheumatol. 2017;69:1127-30.

6. Courties A, Sellam J, Berenbaum F. Metabolic syndrome-associated osteoarthritis. Curr Opin Rheumatol. 2017;29:214-22.

7. Li S, Felson DT. What Is the Evidence to Support the Association Between Metabolic Syndrome and Osteoarthritis? A Systematic Review. Arthritis Care Res. 2019;71:875-84.

8. Obesity: preventing and managing the global epidemic. Report of a WHO consultation. World Health Organ Tech Rep Ser. 2000;894:i-xii, 1-253.

9. Thijssen E, van Caam A, van der Kraan PM. Obesity and osteoarthritis, more than just wear and tear: pivotal roles for inflamed adipose tissue and dyslipidaemia in obesity-induced osteoarthritis. Rheumatol Oxf. 2015;54:588-600.

10. Schwarz S, Mrosewski I, Silawal S, Schulze-Tanzil G. The interrelation of osteoarthritis and diabetes mellitus: considering the potential role of interleukin-10 and in vitro models for further analysis. Inflamm Res. 2018;67:285-300.

11. Buettner R, Scholmerich J, Bollheimer LC. High-fat diets: modeling the metabolic disorders of human obesity in rodents. Obes Silver Spring. 2007;15:798-808.

12. Mulder P, Morrison MC, Wielinga PY, van Duyvenvoorde W, Kooistra T, Kleemann R. Surgical removal of inflamed epididymal white adipose tissue attenuates the development of non-alcoholic steatohepatitis in obesity. Int J Obes. 2016;40:675-84. 
13. Wang CY, Liao JK. A mouse model of diet-induced obesity and insulin resistance. Methods Mol Biol. 2012;821:421-33.

14. Kozijn AE, Gierman LM, van der Ham F, Mulder P, Morrison MC, Kuhnast S, et al. Variable cartilage degradation in mice with diet-induced metabolic dysfunction: food for thought. Osteoarthritis Cartilage. 2018;26:95-107.

15. Glasson SS, Blanchet TJ, Morris EA. The surgical destabilization of the medial meniscus (DMM) model of osteoarthritis in the 129/SvEv mouse. Osteoarthritis Cartilage. 2007;15:1061-9.

16. Mooney R, Sampson E, Lerea J, Rosier R, Zuscik M. High-fat diet accelerates progression of osteoarthritis after meniscal/ligamentous injury. Arthritis Res Ther. 2011;13:R198.

17. Araujo AC, Wheelock CE, Haeggstrom JZ. The Eicosanoids, Redox-Regulated Lipid Mediators in Immunometabolic Disorders. Antioxid Redox Signal. 2018;29:275-96.

18. Dal-Secco D, Wang J, Zeng Z, Kolaczkowska E, Wong CH, Petri B, et al. A dynamic spectrum of monocytes arising from the in situ reprogramming of CCR2+ monocytes at a site of sterile injury. $J$ Exp Med. 2015;212:447-56.

19. Urban $\mathrm{H}$, Little $\mathrm{CB}$. The role of fat and inflammation in the pathogenesis and management of osteoarthritis. Rheumatol Oxf. 2018;57 suppl_4:iv10-21.

20. Glasson SS, Chambers MG, Van Den Berg WB, Little CB. The OARSI histopathology initiative recommendations for histological assessments of osteoarthritis in the mouse. Osteoarthritis Cartilage. 2010;18 Suppl 3:S17-23.

21. Kozijn AE, Tartjiono MT, Ravipati S, van der Ham F, Barrett DA, Mastbergen SC, et al. Human Creactive protein aggravates osteoarthritis development in mice on a high-fat diet. Osteoarthritis Cartilage. 2019;27:118-28.

22. Gabbs M, Leng S, Devassy JG, Monirujjaman M, Aukema HM. Advances in Our Understanding of Oxylipins Derived from Dietary PUFAs. Adv Nutr. 2015;6:513-40.

23. Guilliams M, Mildner A, Yona S. Developmental and Functional Heterogeneity of Monocytes. Immunity. 2018;49:595-613.

24. Ziegler-Heitbrock L, Ancuta P, Crowe S, Dalod M, Grau V, Hart DN, et al. Nomenclature of monocytes and dendritic cells in blood. Blood. 2010;116:e74-80.

25. Louer CR, Furman BD, Huebner JL, Kraus VB, Olson SA, Guilak F. Diet-induced obesity significantly increases the severity of posttraumatic arthritis in mice. Arthritis Rheum. 2012;64:3220-30.

26. Messier SP. Obesity and osteoarthritis: disease genesis and nonpharmacologic weight management. Rheum Clin North Am. 2008;34:713-29.

27. Funk CD. Prostaglandins and leukotrienes: advances in eicosanoid biology. Science. 2001;294:1871-5.

28. Zivkovic AM, Telis N, German JB, Hammock BD. Dietary omega-3 fatty acids aid in the modulation of inflammation and metabolic health. Calif Agric. 2011;65:106-11. 
29. de Visser HM, Mastbergen SC, Ravipati S, Welsing PMJ, Pinto FC, Lafeber F, et al. Local and systemic inflammatory lipid profiling in a rat model of osteoarthritis with metabolic dysregulation. PLoS One. 2018;13:e0196308.

30. Richardson D, Pearson R, Kurian N, Latif ML, Garle M, Barrett D, et al. Characterisation of the cannabinoid receptor system in synovial tissue and fluid in patients with osteoarthritis and rheumatoid arthritis. Arthritis Res Ther. 2008;10:R43.

31. Gierman LM, Wopereis S, van El B, Verheij ER, Werff-van der Vat BJ, Bastiaansen-Jenniskens YM, et al. Metabolic profiling reveals differences in concentrations of oxylipins and fatty acids secreted by the infrapatellar fat pad of donors with end-stage osteoarthritis and normal donors. Arthritis Rheum. 2013;65:2606-14.

32. Korotkova M, Jakobsson P-J. Persisting eicosanoid pathways in rheumatic diseases. Nat Rev Rheumatol. 2014;10:229-41.

33. Laufer S. Role of eicosanoids in structural degradation in osteoarthritis. Curr Opin Rheumatol. 2003;15:623-7.

34. Zha W, Edin ML, Vendrov KC, Schuck RN, Lih FB, Jat JL, et al. Functional characterization of cytochrome P450-derived epoxyeicosatrienoic acids in adipogenesis and obesity. J Lipid Res. 2014;55:2124-36.

35. Wang W, Yang J, Qi W, Yang H, Wang C, Tan B, et al. Lipidomic profiling of high-fat diet-induced obesity in mice: Importance of cytochrome P450-derived fatty acid epoxides. Obes Silver Spring. 2017;25:132-40.

36. Wang W, Yang J, Zhang J, Wang Y, Hwang SH, Qi W, et al. Lipidomic profiling reveals soluble epoxide hydrolase as a therapeutic target of obesity-induced colonic inflammation. Proc Natl Acad Sci U A. 2018;115:5283-8.

37. Iyer A, Kauter K, Alam MA, Hwang SH, Morisseau C, Hammock BD, et al. Pharmacological inhibition of soluble epoxide hydrolase ameliorates diet-induced metabolic syndrome in rats. Exp Diabetes Res. 2012;2012:758614.

38. Liu Y, Dang H, Li D, Pang W, Hammock BD, Zhu Y. Inhibition of soluble epoxide hydrolase attenuates high-fat-diet-induced hepatic steatosis by reduced systemic inflammatory status in mice. PLoS One. 2012;7:e39165.

39. Fernandes GS, Valdes AM. Cardiovascular disease and osteoarthritis: common pathways and patient outcomes. Eur J Clin Invest. 2015;45:405-14.

40. Theken KN, Schuck RN, Edin ML, Tran B, Ellis K, Bass A, et al. Evaluation of cytochrome P450-derived eicosanoids in humans with stable atherosclerotic cardiovascular disease. Atherosclerosis. 2012;222:530-6.

41. Valdes AM, Ravipati S, Pousinis P, Menni C, Mangino M, Abhishek A, et al. Omega-6 oxylipins generated by soluble epoxide hydrolase are associated with knee osteoarthritis. J Lipid Res. 2018;59:1763-70. 
42. Kundu S, Roome T, Bhattacharjee A, Carnevale KA, Yakubenko VP, Zhang R, et al. Metabolic products of soluble epoxide hydrolase are essential for monocyte chemotaxis to MCP-1 in vitro and in vivo. J Lipid Res. 2013;54:436-47.

43. Raghu $\mathrm{H}$, Lepus $\mathrm{CM}$, Wang $\mathrm{Q}$, Wong $\mathrm{HH}$, Lingampalli $\mathrm{N}$, Oliviero F, et al. CCL2/CCR2, but not CCL5/CCR5, mediates monocyte recruitment, inflammation and cartilage destruction in osteoarthritis. Ann Rheum Dis. 2017;76:914-22.

44. Sunderkotter C, Nikolic T, Dillon MJ, Van Rooijen N, Stehling M, Drevets DA, et al. Subpopulations of mouse blood monocytes differ in maturation stage and inflammatory response. J Immunol. 2004;172:4410-7.

45. Wong KL, Yeap WH, Tai JJY, Ong SM, Dang TM, Wong SC. The three human monocyte subsets: implications for health and disease. Immunol Res. 2012;53:41-57.

46. Stansfield BK, Ingram DA. Clinical significance of monocyte heterogeneity. Clin Transl Med. 2015;4:5.

47. Idzkowska E, Eljaszewicz A, Miklasz P, Musial WJ, Tycinska AM, Moniuszko M. The Role of Different Monocyte Subsets in the Pathogenesis of Atherosclerosis and Acute Coronary Syndromes. Scand J Immunol. 2015;82:163-73.

48. Tsukamoto M, Seta N, Yoshimoto K, Suzuki K, Yamaoka K, Takeuchi T. CD14(bright)CD16+ intermediate monocytes are induced by interleukin-10 and positively correlate with disease activity in rheumatoid arthritis. Arthritis Res Ther. 2017;19:28.

49. Loukov D, Karampatos S, Maly MR, Bowdish DME. Monocyte activation is elevated in women with knee-osteoarthritis and associated with inflammation, BMI and pain. Osteoarthritis Cartilage. 2018;26:255-63.

50. Geng S, Chen K, Yuan R, Peng L, Maitra U, Diao N, et al. The persistence of low-grade inflammatory monocytes contributes to aggravated atherosclerosis. Nat Commun. 2016;7:13436.

51. de Jong AJ, Klein-Wieringa IR, Andersen SN, Kwekkeboom JC, Herb-van Toorn L, de Lange-Brokaar BJE, et al. Lack of high BMI-related features in adipocytes and inflammatory cells in the infrapatellar fat pad (IFP). Arthritis Res Ther. 2017;19:186.

52. Daghestani HN, Pieper CF, Kraus VB. Soluble Macrophage Biomarkers Indicate Inflammatory Phenotypes in Patients With Knee Osteoarthritis: Macrophage Markers in OA. Arthritis Rheumatol. 2015;67:956-65.

53. Attur M, Krasnokutsky S, Statnikov A, Samuels J, Li Z, Friese O, et al. Low-grade inflammation in symptomatic knee osteoarthritis: prognostic value of inflammatory plasma lipids and peripheral blood leukocyte biomarkers. Arthritis Rheumatol. 2015;67:2905-15.

54. Scanzello CR, Loeser RF. Editorial: Inflammatory Activity in Symptomatic Knee Osteoarthritis: Not All Inflammation Is Local: EDITORIAL. Arthritis Rheumatol. 2015;67:2797-800.

\section{Tables}


Due to technical limitations, tables 1 and 2 only available as a download in the supplemental files section.

\section{Figures}

\section{Figure 1}

HFD feeding aggravates OA progression at the lateral but not the medial side of the destabilized knee. Representative Safranin-O, Fast Green and Hematoxylin-stained coronal sections showing detailed pictures of the lateral femur condyle and tibial plateau from sham and DMM knee joints of the LFD and HFD group. Original magnification $\times 20$.

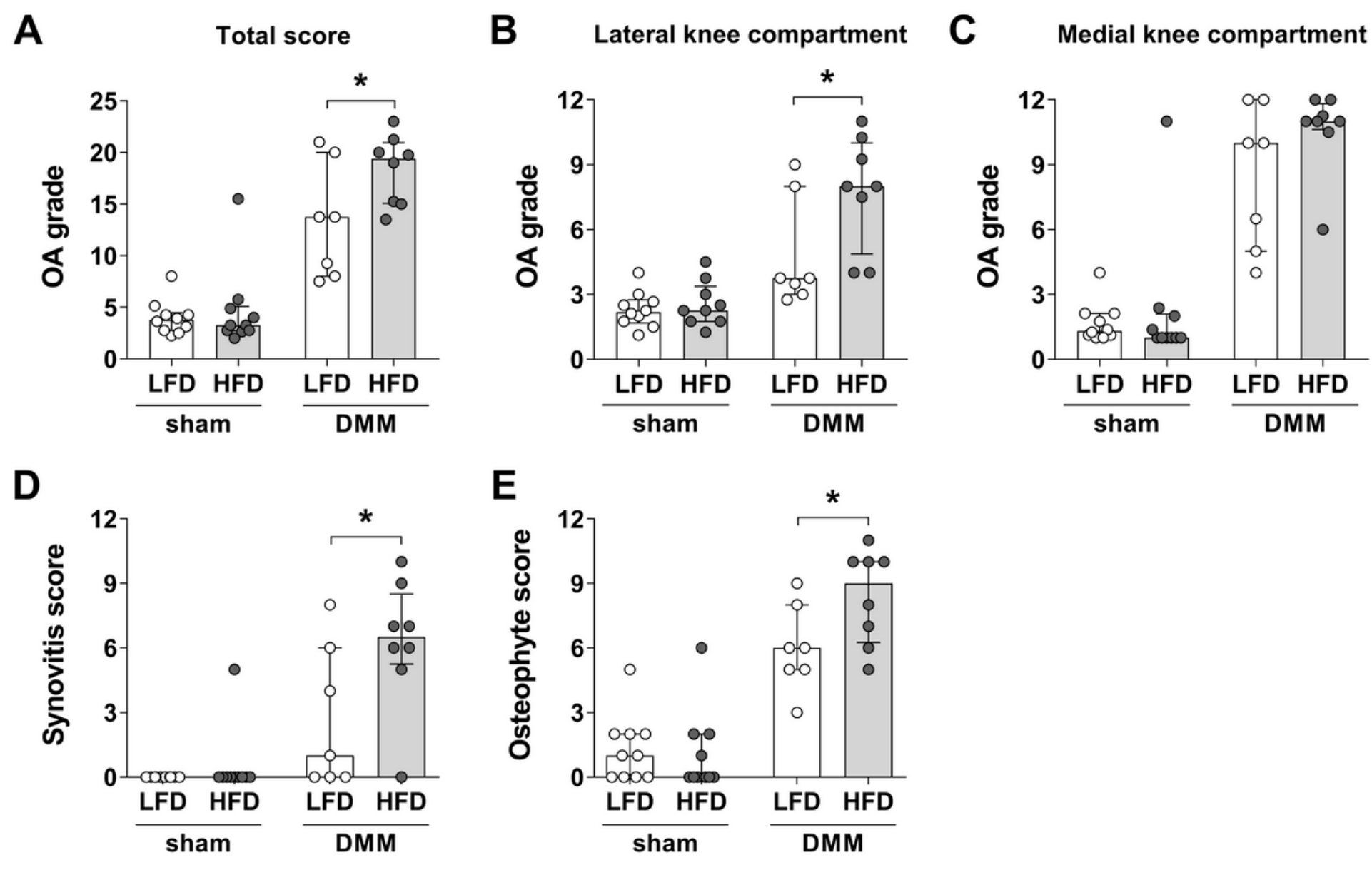

Figure 2

HFD feeding increased cartilage degeneration, osteophyte formation and synovial inflammation. A) Summed histopathological scores for the tibiofemoral knee compartments of the LFD and HFD mice. Cartilage damage was most pronounced at B) the lateral side of the joint in HFD animals, whereas $C$ ) the medial side was severely damaged in both groups. D) Total synovitis scores showing the individual summed score for the tibiofemoral knee compartments for each animal per study group. E) Total 
osteophyte scores showing the individual summed score for the tibiofemoral knee compartments for each animal per study group. Scoring was performed according to OARSI histopathology recommendations for the mouse16. Data are presented as group median (indicated by bars) with interquartile range (error bars). *Statistical significance was set to $p<0.05$.

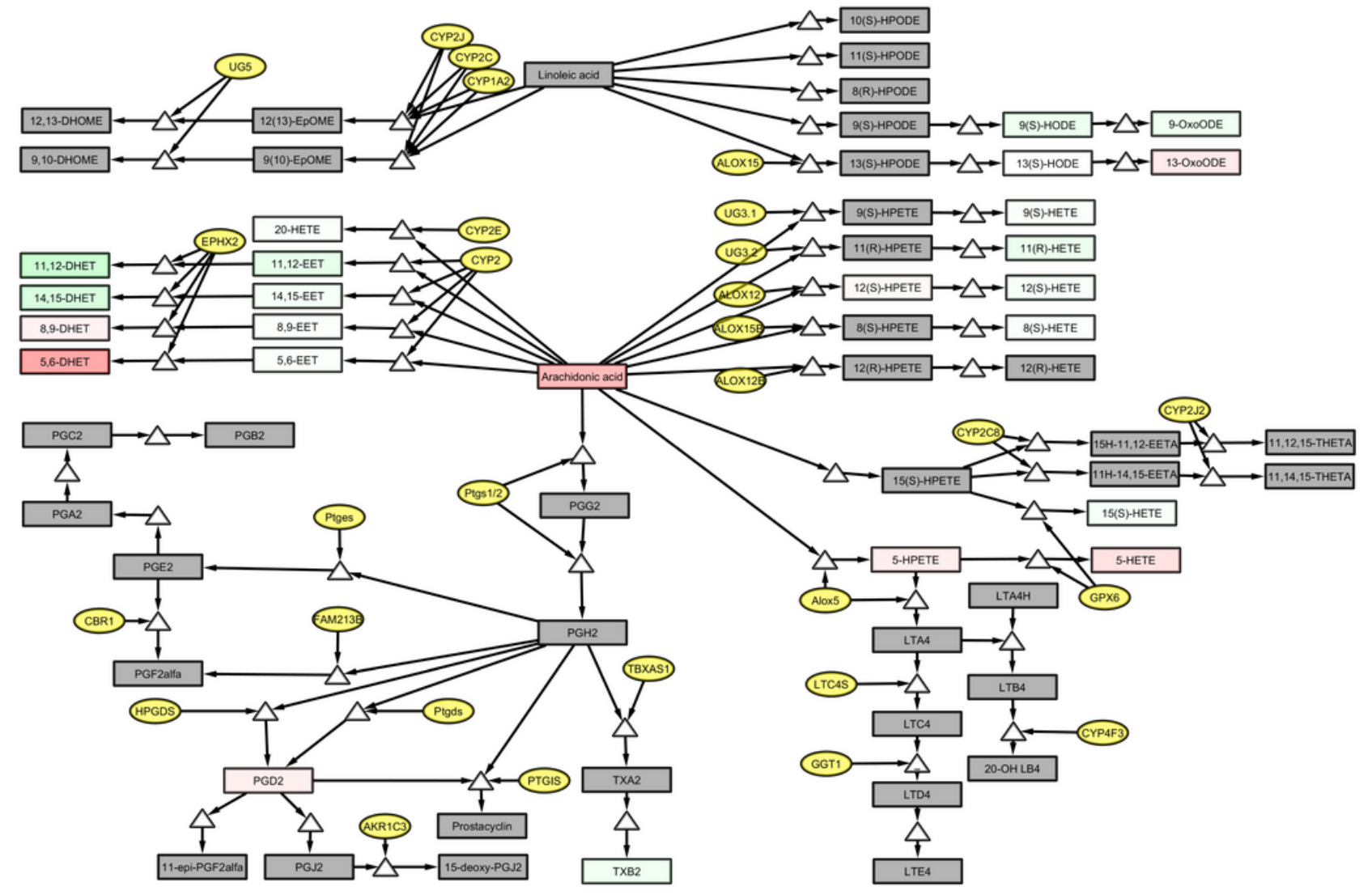

\section{Figure 3}

HFD increased plasma arachidonic acid levels and affected eicosanoid metabolism. Overview of the changes in murine plasma eicosanoid profiles between 0 and 9 weeks after LFD or HFD feeding. Colours indicate the following: grey, not detected or quantified; red, increased concentrations in HFD mice; green, decreased concentrations in HFD mice. Colour intensity reflects the extent to which eicosanoid levels have been elevated or decreased. CYP450, cytochrome P450; COX, cyclooxygenase; LOX, lipoxygenase. 

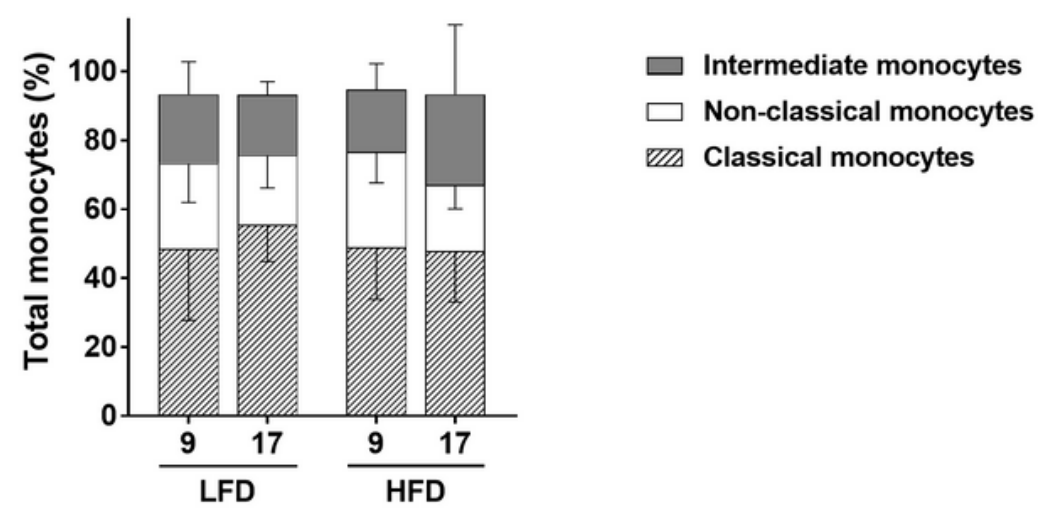

एII

Classical monocytes

B

CD43 ${ }^{\text {low }}$ Ly6C ${ }^{\text {int }}$ monocytes

C

CD11c ${ }^{+}$monocytes
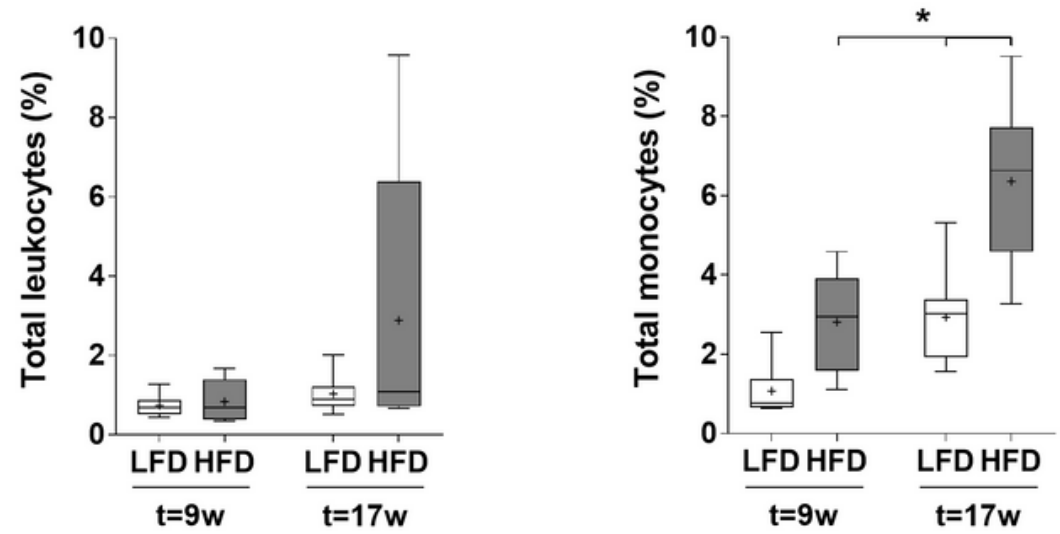

D

Distribution CD11c+ monocytes at $\mathrm{t}=17$ weeks
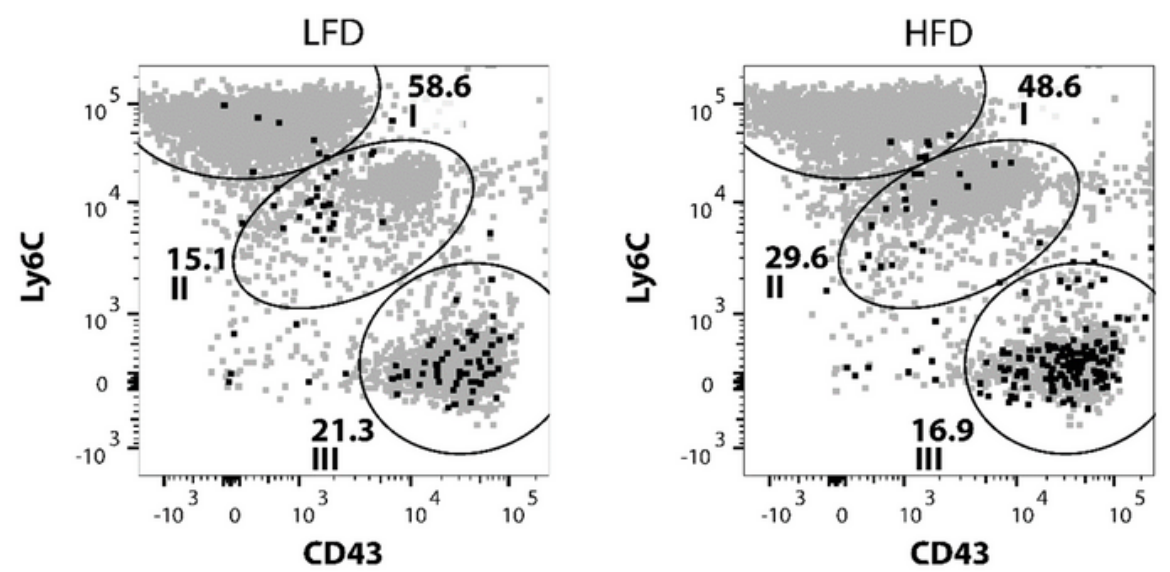

\section{Figure 4}

CD11c-expressing monocytes significantly increased in the HFD group and correlate with osteophytosis and synovial inflammation. A) Peripheral CD115+CD11b+SSC-Alow monocyte subpopulations showed subtle changes in HFD mice compared with LFD mice over time. B) Increased frequency of CD43++Ly6Cint intermediate monocytes plotted as percentage of total leukocytes. C) CD11c-expressing monocytes significantly increased in both diet groups over time, but to a higher degree in the HFD group. 
D) Backgating of CD11 + cells (black dots) to pre-set monocyte gate at $t=17 \mathrm{w}$ revealed an upregulation of CD11c expression by the non-classical CD43++Ly6Clow monocyte subset: I, classical monocytes; II, intermediate monocytes; III, non-classical monocytes. Stacked bars show mean values and standard deviation for each monocyte subset. Box-and-whiskers plots display a statistical summary of the mean (+ within box), median (line within box), interquartile range (box) and the minimum and maximum values (whiskers). *Statistical significance was set to $p<0.05$.
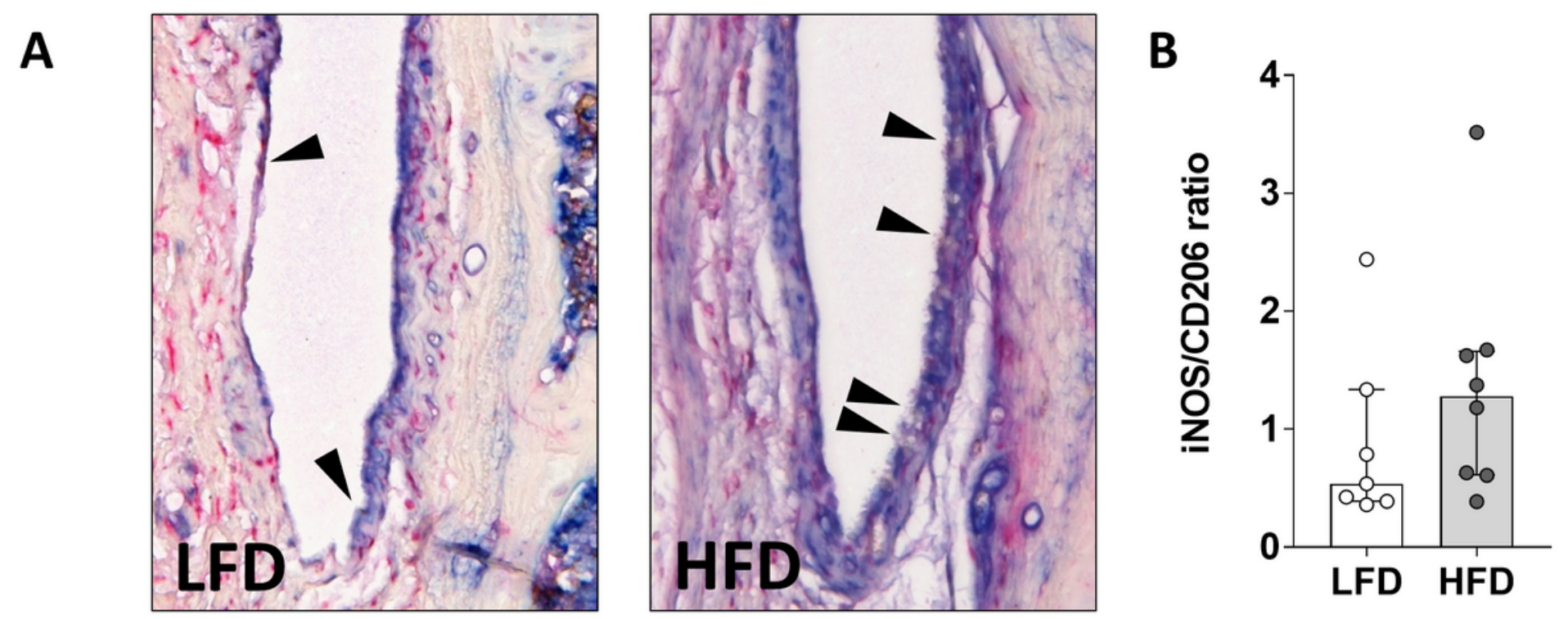

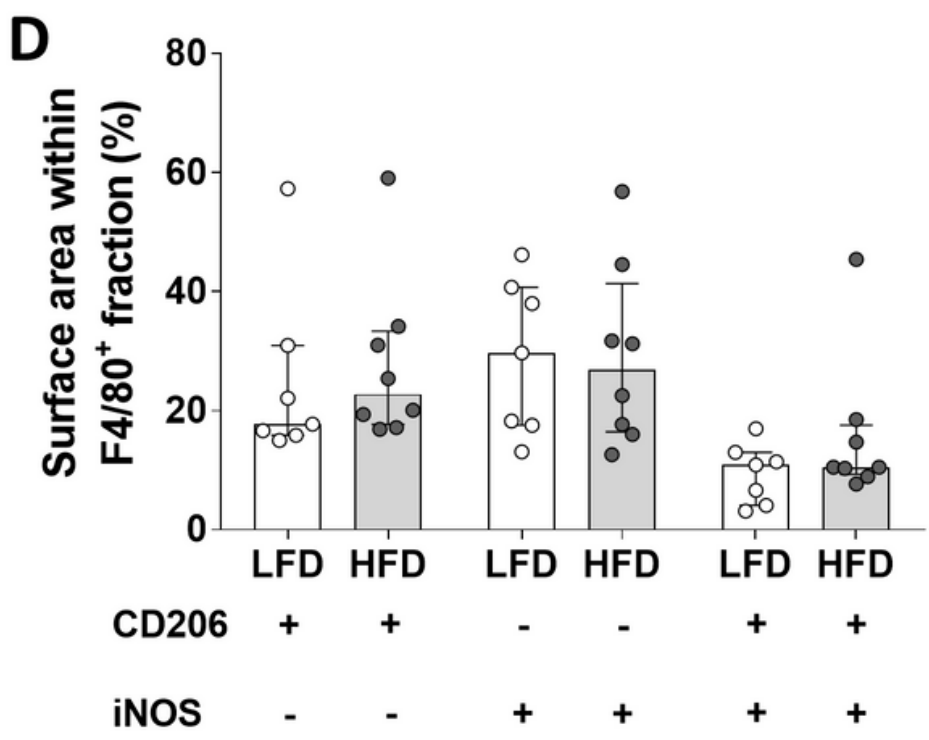

Figure 5

HFD feeding increased macrophage presence but induced no shift in macrophage subset ratio in the synovium. A) Representative images of the lateral patellofemoral synovial lining (ROI) from DMMoperated knees triple stained with pan-macrophage marker F4/80 (yellow, arrowheads), M1 subset marker iNOS (blue) and M2 subset marker CD206 (red). Original magnification $\times 40$. B) Ratio between total iNOS and total CD206 percentage positivity was not significantly different between both groups. C) Percentage marker positivity within the ROI of LFD and HFD mice. D) Percentage CD206 and/or iNOS 
positivity within $\mathrm{F} 4 / 80+$ fraction shows no diet-induced shift in macrophage phenotypes. Data are presented as group median (indicated by bars) with interquartile range (error bars).

\section{Supplementary Files}

This is a list of supplementary files associated with this preprint. Click to download.

- SupplementalFigure1.png

- KozijnetalNC3RsARRIVEChecklist.pdf

- SupplementalFile.pdf

- SupplementalFigure2.png

- Tables.pdf 